\title{
Reducing Transmitted Vibration Using Delayed Hysteretic Suspension
}

\author{
Lahcen Mokni and Mohamed Belhaq \\ Laboratory of Mechanics, University Hassan II, Casablanca, Morocco \\ Correspondence should be addressed to Mohamed Belhaq, mbelhaq@yahoo.fr \\ Received 19 May 2011; Accepted 19 September 2011 \\ Academic Editor: Marc Asselineau
}

Copyright ( $) 2011$ L. Mokni and M. Belhaq. This is an open access article distributed under the Creative Commons Attribution License, which permits unrestricted use, distribution, and reproduction in any medium, provided the original work is properly cited.

Previous numerical and experimental works show that time delay technique is efficient to reduce transmissibility of vibration in a single pneumatic chamber by controlling the pressure in the chamber. The present work develops an analytical study to demonstrate the effectiveness of such a technique in reducing transmitted vibrations. A quarter-car model is considered and delayed hysteretic suspension is introduced in the system. Analytical predictions based on perturbation analysis show that a delayed hysteretic suspension enhances vibration isolation comparing to the case where the nonlinear damping is delay-independent.

\section{Introduction}

Various strategies for reducing transmitted vibrations have been proposed; see, for instance, [1-5] and references therein. A standard technique using linear viscous damping in the vibration isolation device reduces the transmissibility near the resonance but increases it elsewhere. To enhance vibration isolation in the whole frequency range, cubic nonlinear viscous damping has been successfully introduced [3]. It was shown, in the case of a single degree of freedom (sdof) spring damper system, that cubic nonlinear damping can effectively produce an ideal vibration isolation in the whole frequency range [4]. Recently, a strategy based on adding a nonlinear parametric time-dependent viscous damping to the basic cubic nonlinear damper has been proposed [5]. This method significantly enhances vibration isolation comparing to the case where the nonlinear damping is time-independent $[3,4]$. Specifically, it was reported that increasing the amplitude of the parametric damping enhances substantially the vibration isolation over the whole frequency range.

On the other hand, time delay control (TDC) $[6,7]$ was applied to a pneumatic isolator to enhance the isolation performance by controlling the pressure in chamber in the low frequency range [8]. Effectiveness of this active control technique in enhancement of transmissibility performance was demonstrated using simulation as well as experiments testing in the case of a single pneumatic chamber.

In the present paper, we use a delayed hysteretic suspension in the system and we examine its influence on vibration isolation. In this TDC technique, the values of all the state variables and their first derivatives have to be provided by some means.

To achieve our analysis, we implement the multiple scales method [9] on the equation of motion to derive the corresponding modulation equations and we examine the steady state solutions of this modulation equation to obtain indications on transmissibility (TR) versus the system parameters.

\section{Equation of Motion}

A representative model of a suspension system with nonlinear stiffness, nonlinear viscous damping, and delayed hysteretic suspension under external excitation is proposed. 


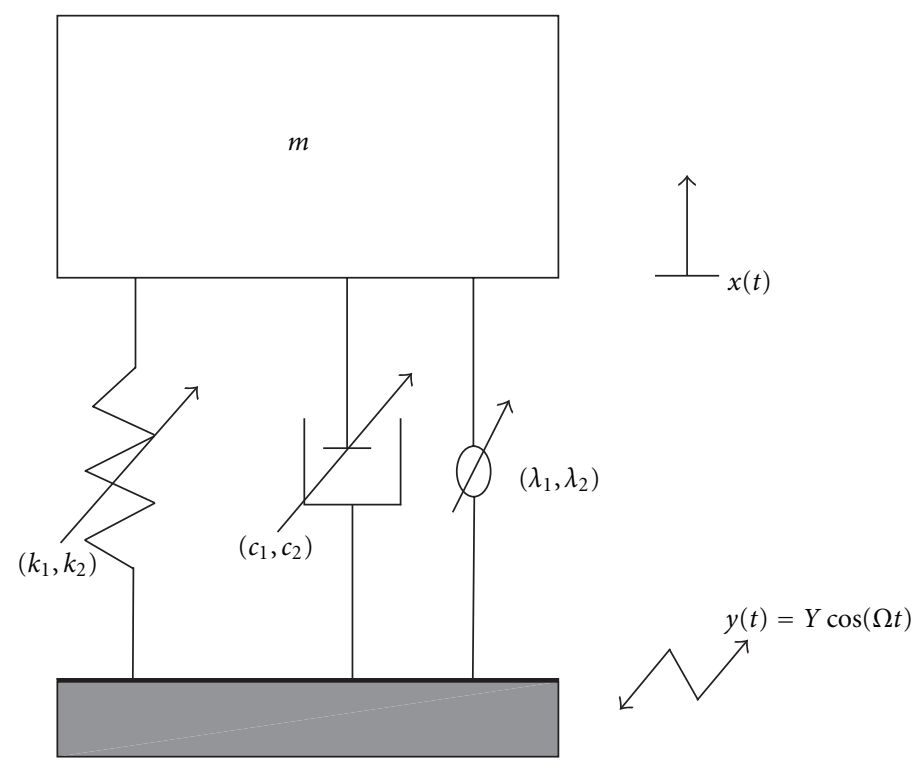

Figure 1: Schematic diagram of pneumatic vibration isolator under ground excitation.

It consists of a sdof model, as shown in Figure 1, described by

$$
\begin{aligned}
\ddot{X}+\omega^{2} X & +B_{1} X^{3}+\left(B_{2} \dot{X}+B_{3} \dot{X}^{3}\right) \\
= & -g+Y \Omega^{2} \cos \Omega t+\lambda_{1} \dot{X}(t-\tau) \\
& +\lambda_{2}(\dot{X}(t-\tau))^{3},
\end{aligned}
$$

where $\omega^{2}=\left(k_{1} / m\right), B_{1}=\left(k_{2} / m\right), B_{2}=\left(c_{1} / m\right)$, and $B_{3}=\left(c_{2} / m\right)$. In the case of a single pneumatic chamber, as shown in Figure $1, m$ is the body mass, $k_{1}$ and $k_{2}$ are the linear and the nonlinear stiffness of vehicle suspension, $c_{1}$ and $c_{2}$ are the linear and the nonlinear equivalent viscous damping, $g$ is the acceleration gravity, and $X$ is the relative vertical displacement of the mass. The parameters $Y$ and $\Omega$ denote, respectively, the amplitude and the frequency of the external excitation, while $\lambda_{1}$ and $\lambda_{2}$ denote the gains of the linear- and the nonlinear-delayed viscous damping, and $\tau$ is the time delay. This delayed viscous damping can be practically implemented using delayed nonlinear dampers based on magnetorheological fluid.

It is worthy to notice that the particular case of linear stiffness $\left(B_{1}=0\right)$ and undelayed state feedback $\left(\lambda_{1}=\lambda_{2}=0\right)$ was studied in [3], while the case of $B_{1} \neq 0, \lambda_{1}=\lambda_{2}=$ 0 , and time-dependent (parametric) damping was treated in [5]. It was demonstrated that adding nonlinear parametric damping to the basic nonlinear damping enhances significantly the vibration isolation [5]. The purpose of the present work is to study the effect of delayed nonlinear damping on vibration isolation of system (1). Note also that a similar delayed nonlinear system to (1) was investigated near primary resonances [10]. Attention was focused on performing an approach to analyze the dynamic of the system with arbitrarily large gains. Note also that a delayed feedback was used to quench undesirable vibrations in a van der Pol type system [11].

\section{Frequency Response and Transmissibility}

To obtain the frequency-response equation and TR, we perform a perturbation method. Introducing a bookkeeping parameter $\epsilon$ and scaling $Y=\epsilon \tilde{Y}, B_{1}=\epsilon \widetilde{B}_{1}, B_{2}=\epsilon \widetilde{B}_{2}, B_{3}=$ $\epsilon \widetilde{B}_{3}, \lambda_{1}=\epsilon \tilde{\lambda}_{1}$, and $\lambda_{2}=\epsilon \tilde{\lambda}_{2}$, (1) reads

$$
\begin{aligned}
\ddot{z}+\omega^{2} z=-g+\epsilon[ & \tilde{Y} \Omega^{2} \cos \Omega t-\widetilde{B}_{1} z^{3}-\widetilde{B}_{2} \dot{z}-\widetilde{B}_{3} \dot{z}^{3} \\
& \left.+\tilde{\lambda}_{1} \dot{z}(t-\tau)+\widetilde{\lambda}_{2}(\dot{z}(t-\tau))^{3}\right] .
\end{aligned}
$$

Using the multiple scales technique [9], a two-scale expansion of the solution is sought in the form

$$
X(t)=z_{0}\left(T_{0}, T_{1}\right)+\epsilon z_{1}\left(T_{0}, T_{1}\right)+O\left(\epsilon^{2}\right),
$$

where $T_{i}=\epsilon^{i} t$. In terms of the variables $T_{i}$, the time derivatives become $d / d t=D_{0}+\epsilon D_{1}+O\left(\epsilon^{2}\right)$ and $d^{2} / d t^{2}=$ $D_{0}^{2}+2 \epsilon D_{0} D_{1}+O\left(\epsilon^{2}\right)$, where $D_{i}^{j}=\left(\partial^{j} / \partial^{j} T_{i}\right)$. Substituting (3) into (2), we obtain

$$
\begin{aligned}
& \left(D_{0}^{2}+2 \epsilon D_{0} D_{1}\right)\left(z_{0}+\epsilon z_{1}\right)+\omega^{2}\left(z_{0}+\epsilon z_{1}\right) \\
& =-g+\epsilon\left[\tilde{Y} \Omega^{2} \cos (\Omega t)-\widetilde{B}_{2}\left(D_{0}+\epsilon D_{1}\right)\left(z_{0}+\epsilon z_{1}\right)\right. \\
& -\widetilde{B}_{1}\left(z_{0}+\epsilon z_{1}\right)^{3}-\widetilde{B}_{3}\left(\left(D_{0}+\epsilon D_{1}\right)\left(z_{0}+\epsilon z_{1}\right)\right)^{3} \\
& +\tilde{\lambda}_{1}\left(D_{0}+\epsilon D_{1}\right)\left(z_{0}(t-\tau)+\epsilon z_{1}(t-\tau)\right) \\
& \left.+\tilde{\lambda}_{2}\left(\left(D_{0}+\epsilon D_{1}\right)\left(z_{0}(t-\tau)+\epsilon z_{1}(t-\tau)\right)\right)^{3}\right]
\end{aligned}
$$


and equating coefficients of the same power of $\epsilon$, we obtain at different orders

$$
\begin{gathered}
D_{0}^{2} z_{0}+\omega^{2} z_{0}=-g, \\
D_{0}^{2} z_{1}+\omega^{2} z_{1}+2 D_{1} D_{0} z_{0} \\
=\widetilde{Y} \Omega^{2} \cos (\Omega t) \\
-\widetilde{B}_{1} z_{0}^{3}-\widetilde{B}_{2} D_{0} z_{0}-\widetilde{B}_{3}\left(D_{0} z_{0}\right)^{3} \\
+\widetilde{\lambda}_{1} D_{0} z_{0}(t-\tau)+\widetilde{\lambda}_{2}\left(D_{0} z_{0}(t-\tau)\right)^{3} .
\end{gathered}
$$

In the case of the principal resonance, that is, $\Omega=\omega+\epsilon \sigma$, where $\sigma$ is a detuning parameter, standard calculations yield the first-order solution:

$$
z(t)=-\frac{g}{\omega^{2}}+a \cos (\Omega t-\gamma)+O(\epsilon),
$$

where the amplitude $a$ and the phase $\gamma$ are given by the modulation equations:

$$
\begin{gathered}
\dot{a}=\frac{\tilde{Y} \Omega^{2}}{2 \omega} \sin (\gamma)-s_{1} a-s_{2} a^{3}, \\
a \dot{\gamma}=\frac{\tilde{Y} \Omega^{2}}{2 \omega} \cos (\gamma)-s_{3} a-s_{4} a^{3} .
\end{gathered}
$$

Here $s_{1}=\left(\widetilde{B}_{2} / 2\right)-\left(\tilde{\lambda}_{1} \cos (\omega \tau) / 2\right), s_{2}=\left(3 \widetilde{B}_{3} \omega^{2} / 8\right)-$ $\left(3 \tilde{\lambda}_{2} \omega^{2} \cos (\omega \tau) / 8\right), s_{3}=\left(3 \widetilde{B}_{1} / 2\right)\left(g^{2} / \omega^{5}\right)-\sigma-\left(\tilde{\lambda}_{1} \sin (\omega \tau) / 2\right)$, and $s_{4}=\left(3 \widetilde{B}_{1} / 8 \omega\right)-\left(3 \tilde{\lambda}_{2} \omega^{2} \sin (\omega \tau) / 8\right)$. Periodic solutions of (2) corresponding to stationary regimes $(\dot{a}=\dot{\gamma}=0)$ of the modulation equations (7) are given by the algebraic equation:

$$
\left(s_{2}^{2}+s_{4}^{2}\right) a^{6}+\left(2 s_{1} s_{2}+2 s_{3} s_{4}\right) a^{4}+\left(s_{1}^{2}+s_{3}^{2}\right) a^{2}-\left(\frac{Y \Omega^{2}}{2 \omega}\right)^{2}=0 .
$$

On the other hand, the relationship between displacement transmissibility and the system parameters is defined by

$$
\mathrm{TR}=\frac{X}{Y}=\sqrt{\left(1+\frac{a}{Y} \cos (\gamma)\right)^{2}+\left(\frac{a}{Y}\right)^{2} \sin ^{2}(\gamma)} .
$$

\section{Influence of Delayed Damping}

The model we consider consists in quarter-car model with softening spring in which $m=240 \mathrm{~kg}, k_{1}=$ $160000 \mathrm{~N} / \mathrm{m}, k_{2}=-30000 \mathrm{~N} / \mathrm{m}^{3}, c_{1}=250 \mathrm{~N} \cdot \mathrm{s} / \mathrm{m}$, and $c_{2}=25 \mathrm{~N} \cdot \mathrm{s}^{3} / \mathrm{m}^{3}$. The amplitude of the excitation frequency is fixed as $Y=0.11$.

Figure 2 illustrates the relative amplitude of motion $a$ versus the frequency $\Omega$, as given by (8) (solid line), and for validation we plot the result obtained by numerical integration using a Rung-Kutta method (circles).

In Figure 3(a) is shown the TR versus $r=\Omega / \omega$ for various feedback gain $\lambda_{1}$ and for $\lambda_{2}=0$. It can be seen in this figure that as $\lambda_{1}$ increases from 0.01 to 15 , the TR reduces. The effect of the feedback gain $\lambda_{2}$ (with $\lambda_{1}=0$ ) on the TR is also

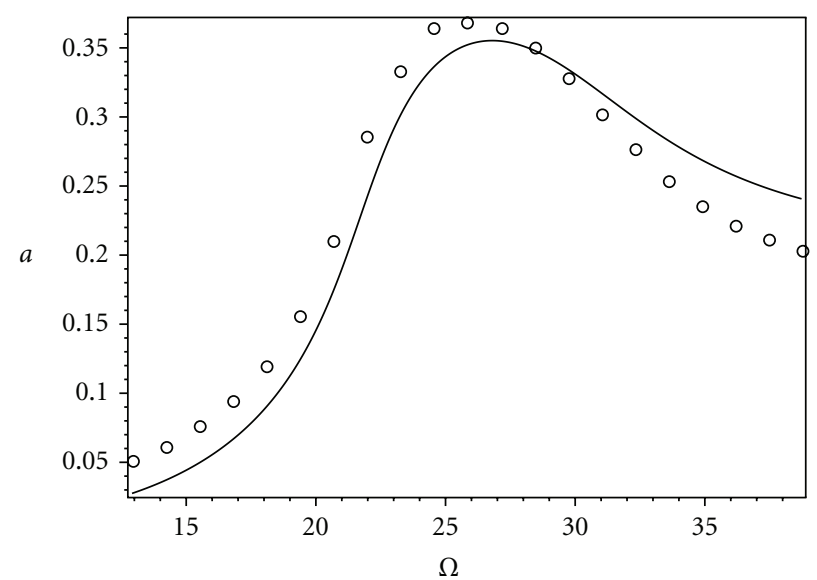

Figure 2: Amplitude $a$ versus $\Omega$, for $\lambda_{1}=0.01, \lambda_{2}=0.01$, and $\tau=$ 0.1 . Analytical prediction: solid line; numerical simulation: circles.

illustrated in Figure 3(b) showing also a decrease of TR as $\lambda_{2}$ increases from 0.01 to 4 . The plots in the figures indicate that to ensure a significant decrease of TR, a small increase in the nonlinear gain $\lambda_{2}$ is sufficient while a larger value of the linear gain $\lambda_{1}$ is necessary to have an equivalent effect.

Figure 4 shows the variation of TR with respect to time delay $\tau$ for different values of the gains $\lambda_{1}$ and $\lambda_{2}$. These plots indicate that increasing the gains causes TR to reduce in repeated periodic intervals of $\tau$. Also, a small increase of $\lambda_{2}\left(\lambda_{2}=0.5\right)$ produces this reduction (Figure $4(\mathrm{~b})$ ), while a larger value of $\lambda_{1}\left(\lambda_{1}=10\right)$ should be introduced to obtain a comparable effect (Figure 4(a)). To validate the analytical predictions (solid lines), we show in Figures 5 and 6 comparisons with the numerical simulations (circles).

\section{Conclusions}

In this work, a strategy based on adding hysteretic nonlinear suspension with time delay to control transmitted vibration is presented. The analytical prediction, based on perturbation method, shows clearly that increasing the amplitude gains of the delayed damping reduces transmitted vibrations to a support structure isolation. This analytical prediction confirms previous numerical and experimental works obtained in the case of a single pneumatic chamber [8]. The results revealed that the case where only the nonlinear gain is acting improves greatly vibration isolation comparing to the case where only the linear gain is applied. Vibration isolation enhancement can be obtained for a small increase of the nonlinear gain $\lambda_{2}$, while a larger increase of the linear gain $\lambda_{1}$ is required to obtain a comparable effect. It is also shown that for small values of the nonlinear gain, vibration isolation can be reduced in repeated periodic intervals of time delay, whereas larger values of the linear gain is needed to obtain a similar result. The method performed in this work provides an approximate expression of transmissibility relating the parameters of the system, thereby showing the explicit dependence between transmissibility and control parameters which is important from monitoring view point. 


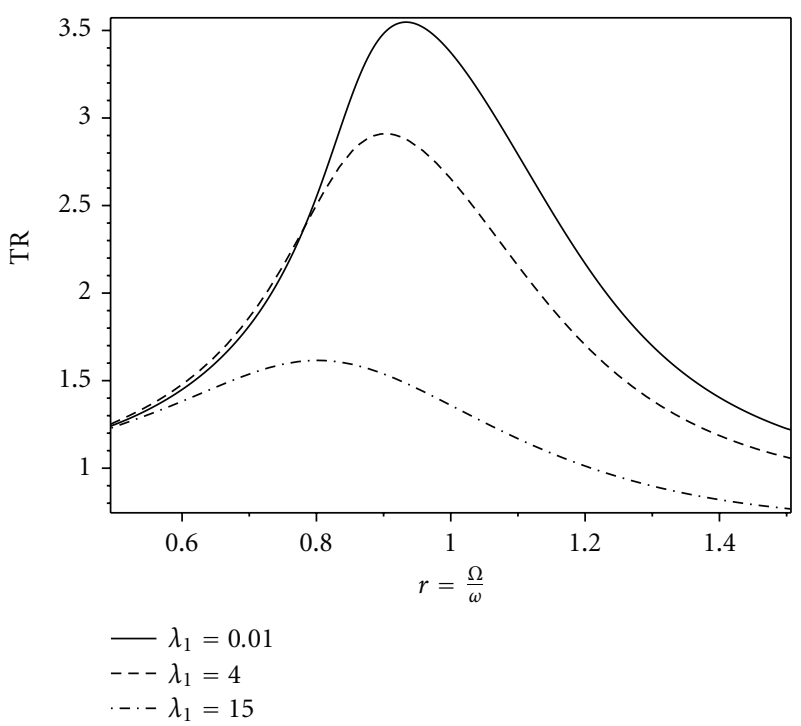

(a)

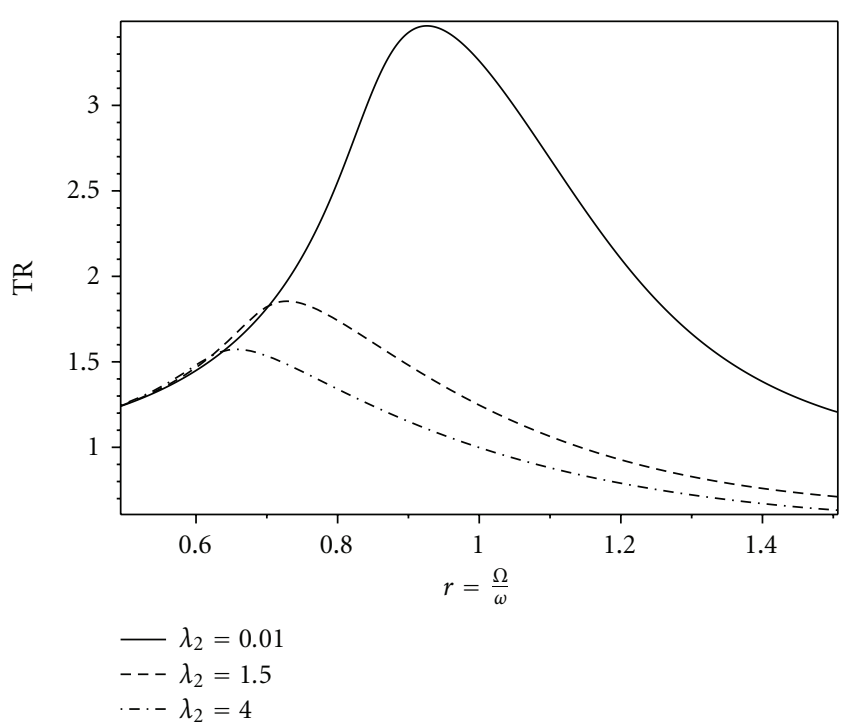

(b)

Figure 3: Transmissibility versus $r$ for $\tau=0.1$. (a) $\lambda_{2}=0$, and (b) $\lambda_{1}=0$.

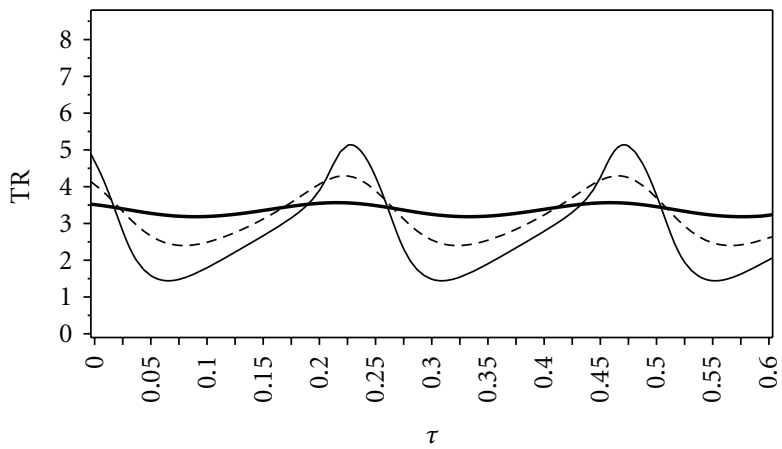

$\begin{aligned}-\lambda_{1} & =1 \\ ---\lambda_{1} & =5\end{aligned}$

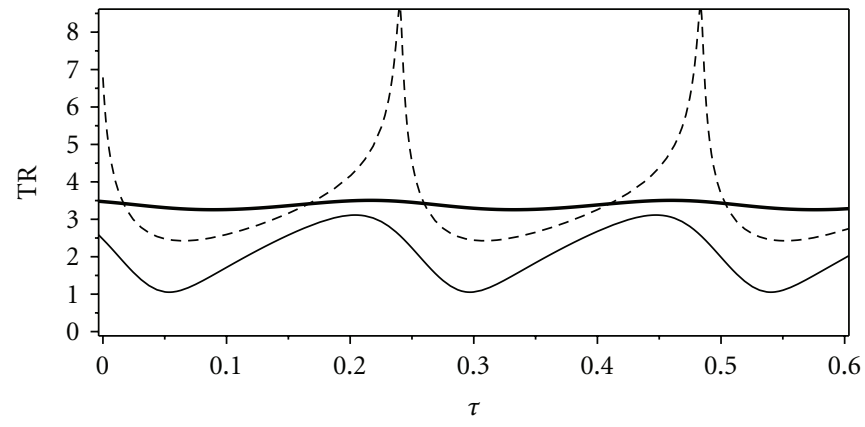

$\begin{aligned}-\lambda_{2} & =0.01 \\ ---\lambda_{2} & =0.1 \\ -\lambda_{2} & =0.5\end{aligned}$

(a)

(b)

Figure 4: Transmissibility versus $\tau$ for $r=1$. (a) $\lambda_{2}=0$, and (b) $\lambda_{1}=0$.

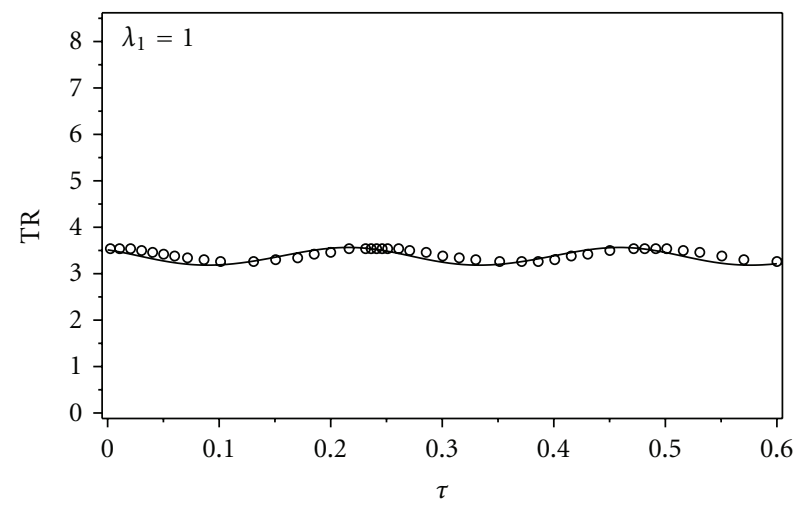

(a)

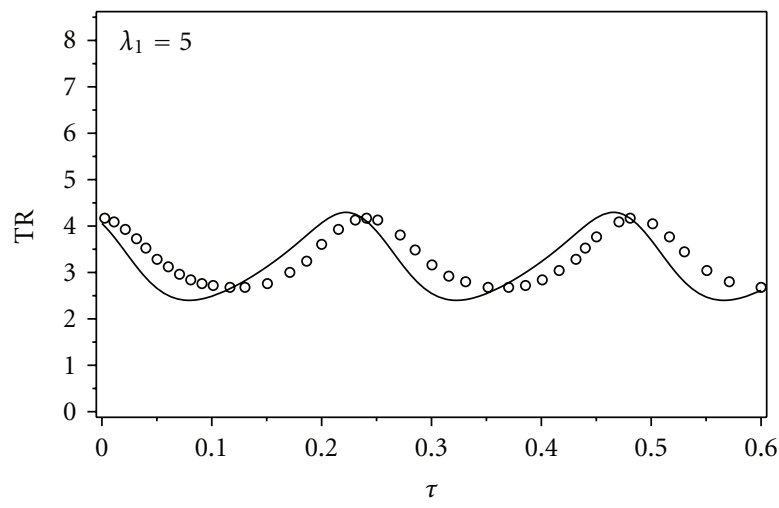

(b)

Figure 5: Transmissibility versus $\tau$ for $r=1$ and $\lambda_{2}=0$. 


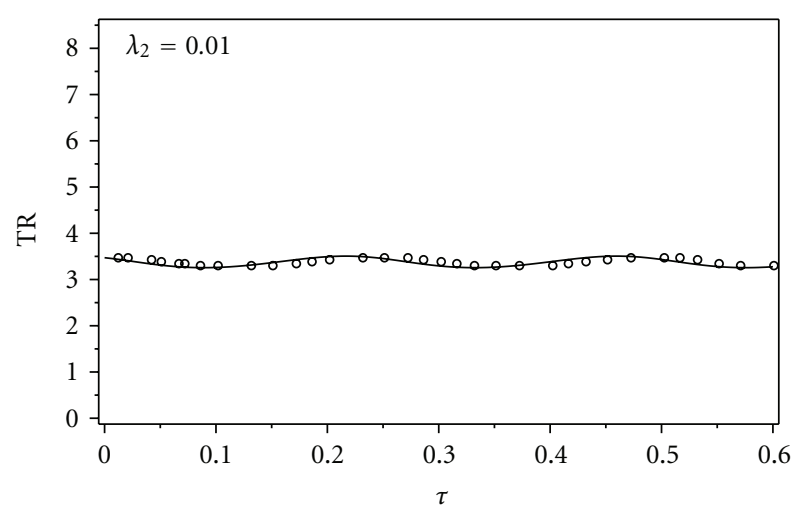

(a)

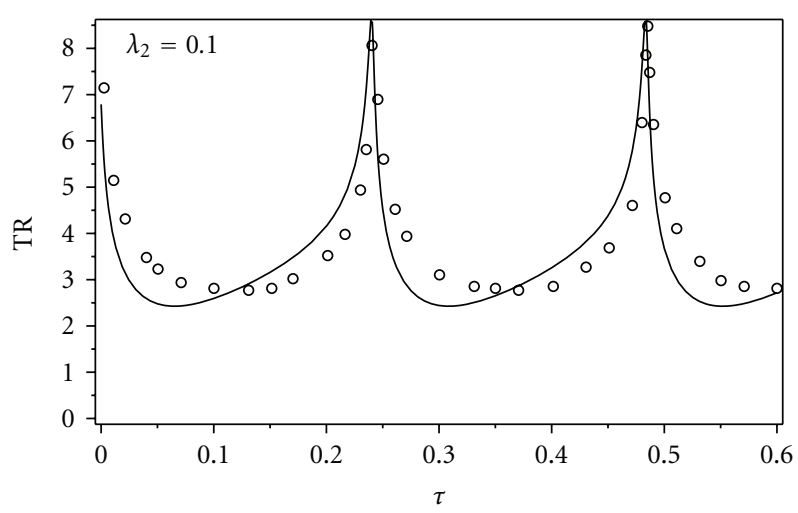

(b)

Figure 6: Transmissibility versus $\tau$ for $r=1$ and $\lambda_{1}=0$.

\section{References}

[1] E. I. Rivin, "Vibration isolation of precision equipment," Precision Engineering, vol. 17, no. 1, pp. 41-56, 1995.

[2] R. A. Ibrahim, "Recent advances in nonlinear passive vibration isolators," Journal of Sound and Vibration, vol. 314, no. 3-5, pp. 371-452, 2008.

[3] Z. Q. Lang, S. A. Billings, G. R. Tomlinson, and R. Yue, "Analytical description of the effects of system nonlinearities on output frequency responses: a case study," Journal of Sound and Vibration, vol. 295, no. 3-5, pp. 584-601, 2006.

[4] Z. Q. Lang, X. J. Jing, S. A. Billings, G. R. Tomlinson, and Z. K. Peng, "Theoretical study of the effects of nonlinear viscous damping on vibration isolation of sdof systems," Journal of Sound and Vibration, vol. 323, no. 1-2, pp. 352-365, 2009.

[5] L. Mokni, M. Belhaq, and F. Lakrad, "Effect of fast parametric viscous damping excitation on vibration isolation in sdof systems," Communications in Nonlinear Science and Numerical Simulation, vol. 16, no. 4, pp. 1720-1724, 2011.

[6] K. Youcef-Toumi and O. Ito, "Time delay controller for systems with unknown dynamics," Journal of Dynamic Systems, Measurement and Control, vol. 112, no. 1, pp. 133-142, 1990.

[7] T. C. Hsia and L. S. Gao, "Robot manipulator control using decentralized linear time-invariant time-delayed joint controllers," in Proceedings of the IEEE International Conference on Robotics and Automation, vol. 3, pp. 2070-2075, Cincinnati, Ohio, USA, May 1990.

[8] Y. H. Shin and K. J. Kim, "Performance enhancement of pneumatic vibration isolation tables in low frequency range by time delay control," Journal of Sound and Vibration, vol. 321, no. 3-5, pp. 537-553, 2009.

[9] A. H. Nayfeh and D. T. Mook, Nonlinear Oscillations, Wiley, New York, NY, USA, 1979.

[10] M. F. Daqaq, K. A. Alhazza, and Y. Qaroush, "On primary resonances of weakly nonlinear delay systems with cubic nonlinearities," Nonlinear Dynamics, vol. 64, pp. 253-277, 2011.

[11] M. K. Suchorsky, S. M. Sah, and R. H. Rand, "Using delay to quench undesirable vibrations," Nonlinear Dynamics, vol. 62, pp. 407-416, 2010. 

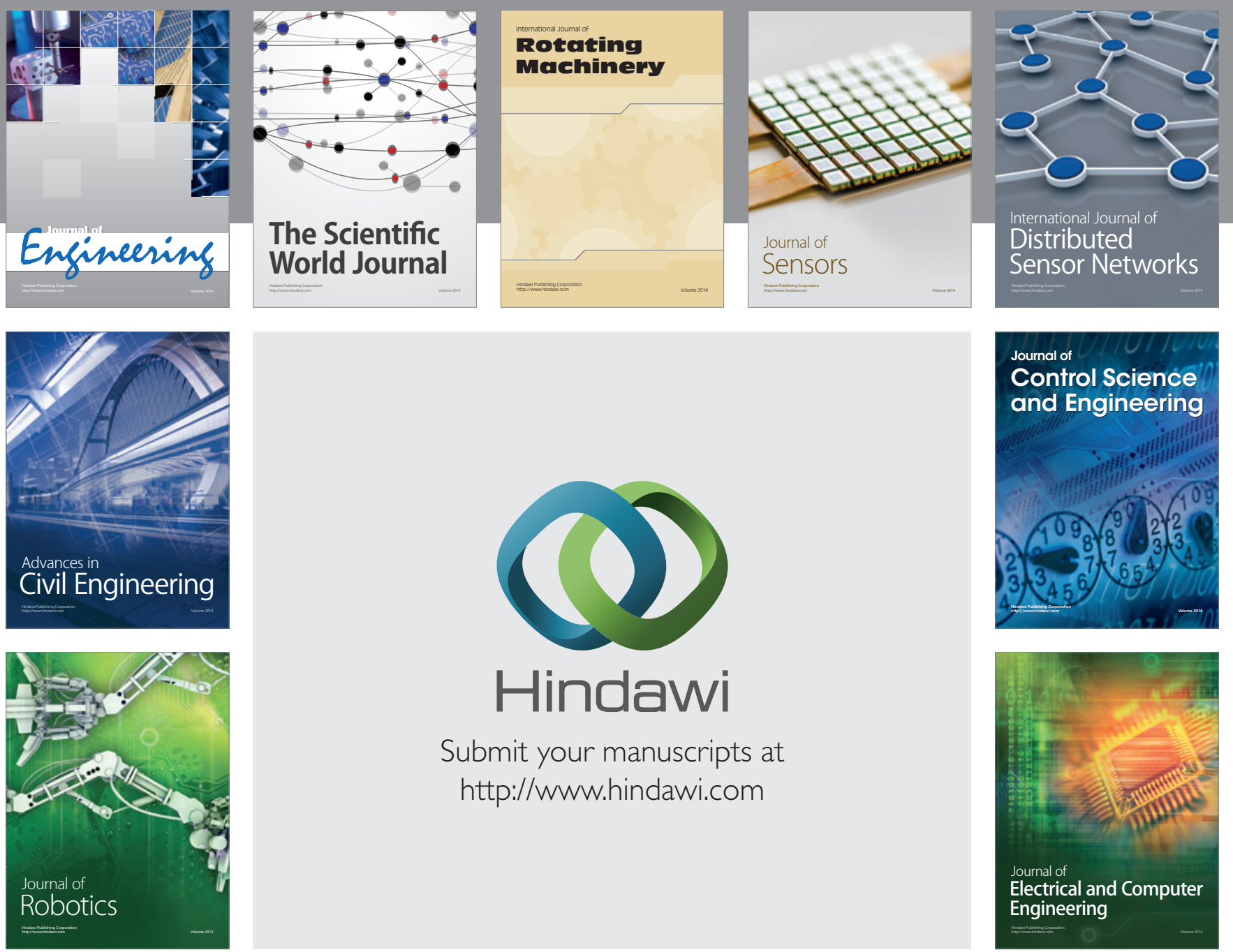

Submit your manuscripts at

http://www.hindawi.com
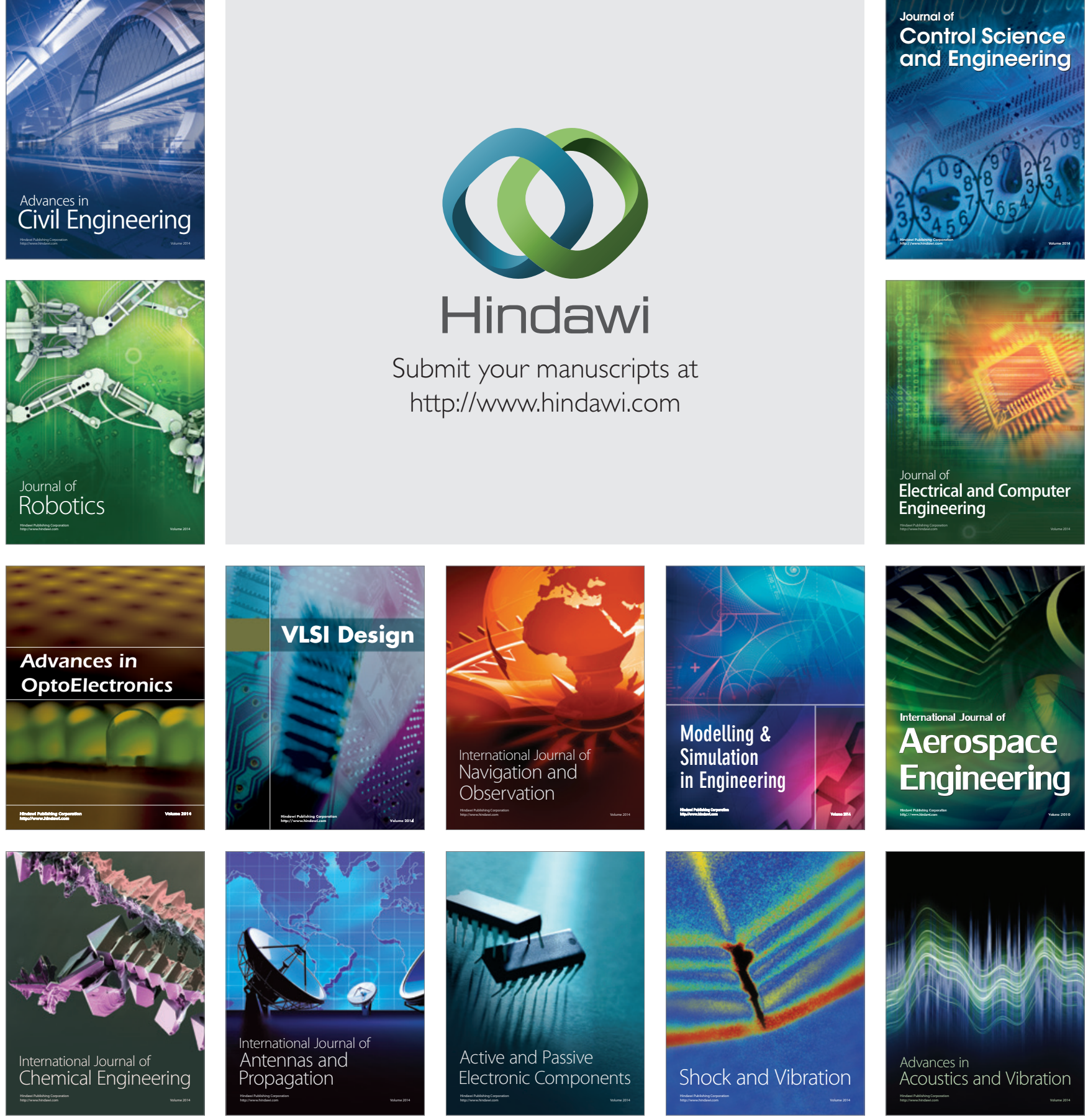